\title{
Benign Salivary Gland Myoepithelioma
}

National Cancer Institute

\section{Source}

National Cancer Institute. Benign Salivary Gland Myoepithelioma. NCI Thesaurus. Code C5978.

A rare benign tumor composed of sheets and islands of spindle, plasmacytoid, epithelioid, and clear cells that exhibit myoepithelial but not ductal differentiation. The average age of patients has been reported as 44 years. Clinically, these tumors present as asymptomatic masses. Discrimination between myoepithelioma and myoepithelial carcinoma is based primarily on the infiltrative growth of the latter. 